\title{
Construction of a Bcl-2-shRNA expression vector and its effect on the mitochondrial apoptosis pathway in SW982 cells
}

\author{
WEIDONG ZHANG ${ }^{1}$, XINJIE XUE ${ }^{1}$ and TENG FU ${ }^{2}$ \\ ${ }^{1}$ Guangming Hospital of Traditional Chinese Medicine, Shanghai 201300, P.R. China; \\ ${ }^{2}$ University of Kansas, Lawrence, KS 66045, USA
}

Received April 11, 2017; Accepted September 19, 2017

DOI: $10.3892 / \mathrm{ijmm} .2017 .3156$

\begin{abstract}
Apoptosis is considered to serve an important role in the pathogenesis of rheumatoid arthritis. The aim of the present study was to construct Bcl-2-short hairpin (sh)RNA expression vectors and transfect them into human synovial sarcoma SW982 cells, in order to screen for an effective interference sequence and analyze the effects of this interference on the expression levels of Bcl-2 and other molecules associated with the mitochondrial apoptosis pathway. Three different shRNAs (Bcl-2-sh1, 2 and 3) were designed according to the human Bcl-2 mRNA target sequence and were transformed into competent DH5a Escherichia coli cells following the construction of an expression vector, which was then transfected into SW982 cells. SW982 cells were grouped into a control group (transfected with a negative control shRNA), and Bcl-2-sh1, Bcl-2-sh2 and Bcl-2-sh3 groups (transfected with Bcl-2-sh1, 2 and 3, respectively). The expression levels of Bcl-2 mRNA were detected using reverse transcription-quantitative PCR (RT-qPCR). Bcl-2-sh1 was identified as the most effective shRNA sequence for interference, and was used for subsequent experiments. The mRNA and protein expression levels of Bcl-2, Bax, CytC and Caspase- 3 were detected in SW982 cells by RT-qPCR and western blotting at various time-points (48 and $72 \mathrm{~h}$ ) following transfection with Bcl-2-sh1, in order to observe the effectiveness of this interference. Compared with the control group, the expression levels of $\mathrm{Bcl}-2$ were decreased, while those of Bax, CytC and Caspase- 3 were increased in Bcl-2-sh1-transfected cells $(\mathrm{P}<0.01)$. The interference effect was greater at $48 \mathrm{~h}$ than at $72 \mathrm{~h}$. In summary, an effective shRNA sequence (Bcl-2-sh1) targeting the Bcl-2 gene was identified from three candidates, and was demonstrated to significantly interfere with the expression of Bcl-2, Bax,
\end{abstract}

Correspondence to: Professor Weidong Zhang, Guangming Hospital of Traditional Chinese Medicine, 43 Dongmen Street, Pudong New District, Shanghai 201300, P.R. China

E-mail: 13937110506@163.com

Key words: Bcl-2, short hairpin RNA, mitochondrial pathway, SW982 cells
CytC and Caspase-3 when transfected into SW982 cells. The interference effect of Bcl-2-sh1 was more pronounced at $48 \mathrm{~h}$ than at $72 \mathrm{~h}$ post-transfection.

\section{Introduction}

Rheumatoid arthritis (RA) is an autoimmune disease that is characterized by chronic progressive arthropathy $(1,2)$. Apoptosis is considered to serve an important role in the pathogenesis of RA; in particular, there is a lack of apoptosis in synovial cells and an excess of apoptosis in cartilage cells $(3,4)$. The mitochondrial signaling pathway is a common apoptotic pathway, in which the Bcl-2 family members function as pro-apoptotic and anti-apoptotic signal transduction factors. When apoptotic activation signals are received, Bax oligomerizes, escaping inhibition by $\mathrm{Bcl}-2$, and is inserted into the mitochondrial membrane; the subsequent changes facilitate the release of CytC into the cytosol, where it interacts with the activating factor Apaf-1 to form a multimeric complex. Caspase-9 recruitment initiates the caspase cascade, which involves the activation of downstream Caspase- 3 and eventually results in apoptosis (Fig. 1) (5-7).

As Bcl-2 is the initiating factor of the mitochondrial pathway, and its transcripts have been found to be highly expressed in the synovial tissues and cells of patients with RA (8), the present study aimed to design and synthesize human Bcl-2-short hairpin (sh)RNA expression vectors and assess their effects. The vectors were transformed into competent DH5a Escherichia coli (a genetically engineered Escherichia coli) cells, and then transfected into the human synovial sarcoma cell line SW982 for screening of an effective interference sequence. The expression levels of molecules associated with the mitochondrial pathway were then detected. The present study provides a theoretical and experimental basis for a potential molecular targeting treatment for RA.

\section{Materials and methods}

Materials. Type I collagenase, Dulbecco's modified Eagle's medium (DMEM)/F12 (glucose-free) and trypsin were purchased from Corning Inc. (Corning, NY, USA). DNA endonuclease enzymes (XhoI and $M l u \mathrm{I})$ were purchased from Shanghai Yu Bo Biological Technology Co., Ltd. (Shanghai, China). Lipofectamine 2000, First Strand cDNA Synthesis kit, 
Table I. The sequences of Bcl-2 pre-short hairpin RNA.

Oligo name $\quad$ Single stranded oligonucleotide sequence $\left(5^{\prime}-3^{\prime}\right)$

Bcl-2 I-F

Bcl-2 I-R

Bcl-2 II-F

Bcl-2 II-R

Bcl-2 III-F

Bcl-2 III-R

\begin{abstract}
CACCCGGGAGATAGTGATGAAGTTTCAAGAGAACTTCATCACTATCTCCCGTTTTTTG AGCTCAAAAAACGGGAGATAGTGATGAAGTTCTCTTGAAACTTCATCACTATCTCCCG CACCTGGATGTTCTGTGCCTGTA TTCAAGAGATACAGGCACAGAACATCCATTTTTTG AGCTCAAAAAATGGATGTTCTGTGCCTGTATCTCTTGAATACAGGCACAGAACATCCA CACCTGTCTTTTGTTGTTGTTCATTCAAGAGATGAACAACAACAAAAGACATTTTTTG AGCTCAAAAAATGTCTTTTGTTGTTGTTCATCTCTTGAATGAACAACAACAAAAGACA
\end{abstract}

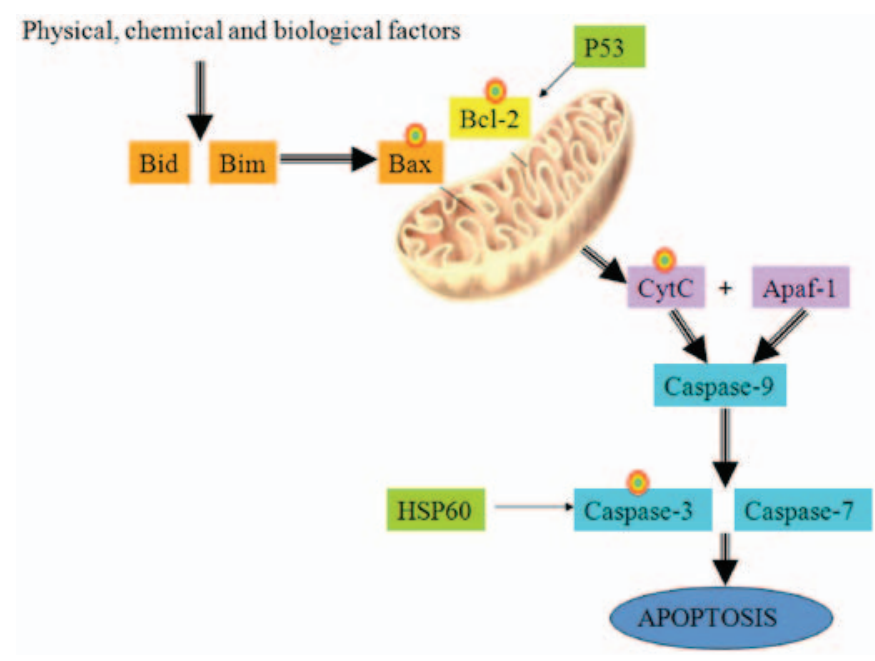

Figure 1. Mitochondrial signaling pathway (28). Source: Chinese doctoral dissertation full text database: Weidong Zhang, The Third Affiliated Hospital of Zhengzhou University, May 2015.

Plasmid Extraction kit, Cell Total RNA Extraction kit, 2X SG Fast qPCR Master Mix and fetal bovine serum (FBS) were purchased from Bio Basic Inc. (Amherst, NY, USA). Human synovial sarcoma SW982 cells were purchased from Bohu Biotechnology Co. (Shanghai, China). Cells between passages 3 and 5 (P3-P5) were used in experiments.

Experiments were carried out in the Laboratory of Molecular Biology at the Bioengineering Biotechnology Company of Shanghai (Shanghai, China) between September and December 2016.

Design and synthesis of Bcl-2-shRNA. Using bioinformatics methods $(9,10)$, the complete sequence of Bcl-2 mRNA (serial number: NM_000633.2) was acquired from GenBank, three sequences were designed for the target gene, and the corresponding sense and antisense oligonucleotides were designed and synthesized (Table I).

Construction of Bcl-2-shRNA interference vector. According to the instructions of the plasmid construction kit (DNA Blunting kit; Takara Bio, Inc., Shiga, Japan; cat. no. 6025), three pairs of shRNAs and a negative control oligonucleotide strand (each $5 \mu \mathrm{l}$ ) were respectively heated $\left(95^{\circ} \mathrm{C}\right)$ for $5 \mathrm{~min}$ in the annealing buffer, and cooled for $20 \mathrm{~min}$ at room temperature. Following the formation of double chains, the oligonucleotides were ligated into the pHAV3.1-shRNA-tGFP vector by T4
DNA ligase. Subsequently, this mixture $(10 \mu \mathrm{l})$ was added to $200 \mu \mathrm{l}$ competent DH5 $\alpha$ E. coli cells (Beijing World Gold Biotech Co., Ltd., Beijing, China) for the transformation step, in which the system was incubated on ice for $30 \mathrm{~min}$, then heatshocked at $42^{\circ} \mathrm{C}$ for $45 \mathrm{sec}$. Subsequently, lysogeny broth (LB) plates with ampicillin were coated with the $E$. coli, and the cells were cultured overnight at $37^{\circ} \mathrm{C}$. Finally, positive clones were selected for the extraction of the DNA plasmids. The positive strains were cultured overnight in LB liquid culture medium, and the plasmids were extracted using the Plasmid Extraction kit, and then, using a double DNA endonuclease (XhoI and $M l u \mathrm{I}$ ) digestion for identification, the plasmids were sent to Invitrogen (Thermo Fisher Scientific, Inc., Waltham, MA, USA) to be sequenced.

Culture and passage of SW982 cells. The SW982 cells were cultured in DMEM-F12 containing 2.5\% FBS and 5\% horse serum, and were placed in an incubator at a temperature of $37^{\circ} \mathrm{C}$ with saturated humidity and $5 \% \mathrm{CO}_{2}$. After the cells had grown to $80 \%$ confluence, they were transferred into a maintenance culture medium (DMEM/F12, $100 \mathrm{nmol} / \mathrm{l}$ dexamethasone and $100 \mathrm{nmol} / \mathrm{l}$ insulin) for 8 days. The day after this, the medium was replaced and the cells were stained with Oil Red O.

Transfection of SW982 cells with the shRNA expression plasmid. In serum-free DMEM-F12 medium, SW982 cells were transfected with the Bcl-2 shRNAs and negative control plasmids using Lipofectamine 2000. SW982 cells were grouped into a control group (transfected with a negative control shRNA), and Bcl-2-sh1, Bcl-2-sh2 and Bcl-2-sh3 groups (transfected with Bcl-2-sh1, 2 and 3, respectively). Following transfection for $6 \mathrm{~h}$, the culture medium was replaced by DMEM-F12 medium containing $10 \%$ FBS and the cells were cultured for a further 48-72 h. The number, intensity and distribution of successfully transfected cells, identified by their expression of green fluorescent protein (GFP), were observed by fluorescence microscopy at different time-points. If the fluorescence intensity was uniform and bright, the transfection efficiency was deemed to be high and the total RNA of the cells was extracted.

Screening for effective interference sequence by reverse transcription-quantitative PCR (RT-qPCR). Total RNA was extracted from the cultured cells using the UN1Q-10 Column TRIzol Total RNA Isolation kit (Sangon Biotech Co., Ltd., Shanghai, China; cat. no. B511321) and was quantified by UV 
Table II. Gene-specific primers used.

Primer sequence $\left(5^{\prime}-3^{\prime}\right)$

\begin{tabular}{llll} 
Gene & \multicolumn{1}{c}{ GenBank ID } & \multicolumn{1}{c}{ Forward } & \multicolumn{1}{c}{ Reverse } \\
\hline Bcl-2 & NM_000633.2 & TTGCCAGCCGGAACCTATG & CGAAGGCGACCAGCAATGATA \\
Bax & NM_001291428.1 & CCCGAGAGGTCTTTTCCGAG & CCAGCCCATGATGGTTCTGAT \\
CytC & NM_018947.5 & TTTGGTTGCACTTACACCGG & GGACGTCCCCACTCTCTAAG \\
Caspase-3 & NM_004346.3 & CATGGAAGCGAATCAATGGACT & CTGTACCAGACCGAGATGTCA \\
$\beta$-actin & NM_001101.3 & CATCCGCAAAGACCTGTACG & CCTGCTTGCTGATCCACATC \\
\hline
\end{tabular}

spectrophotometry (260 nm; NanoDrop ND-100; Thermo Fisher Scientific, Inc., Wilmington, DE, USA). RT was performed with the First Strand cDNA Synthesis kit (Sangon Biotech) and the resultant cDNA was used as the template for qPCR using a Prism 9700 StepOne ${ }^{\mathrm{TM}}$ Real-Time PCR system (Eastwin Life Sciences, Inc., Beijing, China). The primers were synthesized by Sangon Biotech. The thermal cycling conditions were as follows: 1 cycle of $95^{\circ} \mathrm{C}$ for $10 \mathrm{~min}$; and 40 cycles of $95^{\circ} \mathrm{C}$ for $5 \mathrm{sec}$ and $60^{\circ} \mathrm{C}$ for $30 \mathrm{sec}$. The $2^{-\Delta \Delta \mathrm{Ct}}$ method was used to calculate the transcript expression levels relative to those of $\beta$-actin, which served as an internal control (11). The sequence with the highest interference efficiency was selected according to the results of the quantitative detection of Bcl-2 mRNA.

Quantitative measurement of mitochondrial pathway gene expression levels. shRNA transfection, total RNA extraction and RT-qPCR were performed as described above. The primers used for gene-specific amplification are listed in Table II.

Quantitative measurement of mitochondrial pathway protein expression levels using western blotting. Total protein was isolated from the Bcl-2-sh1- and negative control-transfected cells using RIPA lysis buffer (Beyotime Biotech Co., Ltd., Shanghai, China) and subjected to western blot analysis. The protein concentration was determined by BCA assay (BCA assay kit; Beyotime Biotech). The total protein samples $(40 \mu \mathrm{g})$ were resolved by $15 \%$ SDS-PAGE and electro-transferred onto nitrocellulose membranes. Non-specific binding sites were blocked by incubating the membranes at room temperature with $5 \mathrm{X}$ TBS with $10 \%$ BSA. Subsequently, the membranes were probed for mitochondrial pathway proteins through incubation with primary antibodies (all diluted 1:1,000; anti-Bcl-2, ab47489; anti-Bax, ab54829; anti-CytC, ab90529; anti-caspase-3, ab59388; and anti-tubulin, ab6046; all from Abcam, Cambridge, UK) for $60 \mathrm{~min}$ at $37^{\circ} \mathrm{C}$, followed by incubation with the appropriate secondary antibodies (horseradish peroxidase-labeled goat anti-rabbit IgG; Sangon Biotech) for $60 \mathrm{~min}$ at $37^{\circ} \mathrm{C}$. Immunoreactivity was detected by the enhanced chemiluminescence method using an ECL kit (Beyotime Institute of Biotechnology, Haimen, China). Data were obtained from at least three individual experiments performed in triplicate, and the expression levels of the mitochondrial pathway proteins were normalized to those of tubulin.

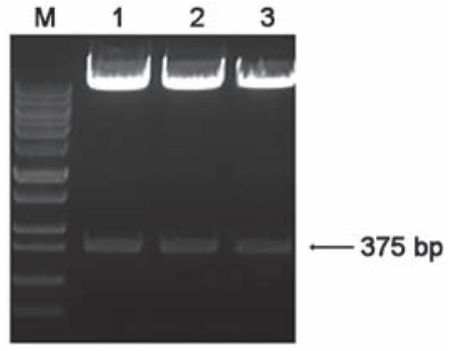

Figure 2. Results of recombinant plasmid electrophoresis following digestion. Lane M, Marker (1-kb DNA Ladder); lane 1, plasmid Bcl-2-sh1 digested by $\mathrm{XhoI}$ and $\mathrm{MluI}$; lane 2, plasmid Bcl-2-sh2 digested by $\mathrm{XhoI}$ and $M l u \mathrm{I}$; lane 3, plasmid Bcl-2-sh3 digested by XhoI and MluI.

Statistical analysis. Statistical analyses were performed with the SPSS software (version 18.0; SPSS, Inc., Chicago, IL, USA). Data are expressed as the mean \pm standard deviation, and inter-group differences were evaluated with a Student's t-test. $\mathrm{P}<0.05$ was considered to indicate a statistically significant difference.

\section{Results}

Construction and identification of Bcl-2 shRNA expression plasmid. Bcl-2-shRNA expression plasmids 1,2 and 3, which were identified by double enzyme digestion and $1 \%$ agarose gel electrophoresis, were synthesized successfully (Fig. 2). The shRNA expression plasmids were confirmed by sequencing analysis; the recombinant plasmids contained shRNA fragments, and the nucleotide sequences of the inserted fragments were complete, and were consistent with the designed sequences (Fig. 3).

Observation of Bcl-2 shRNA expression plasmid-transfected SW982 cells by fluorescence microscopy. Following the transfection of Bcl-2 shRNA expression plasmids into SW982 cells (P3-P5), GFP expression was observed by fluorescence microscopy. GFP expression peaked at $48 \mathrm{~h}$, and the fluorescence intensity in the cytoplasm was uniform and bright, which indicated that the transfection was successful (Fig. 4).

Expression of target gene Bcl-2 and screening for an effective interference sequence. RT-qPCR was used to detect the efficacy of Bcl-2 inhibition in transfected SW982 cells, which revealed that the Bcl-2-sh1, Bcl-2-sh2 and 

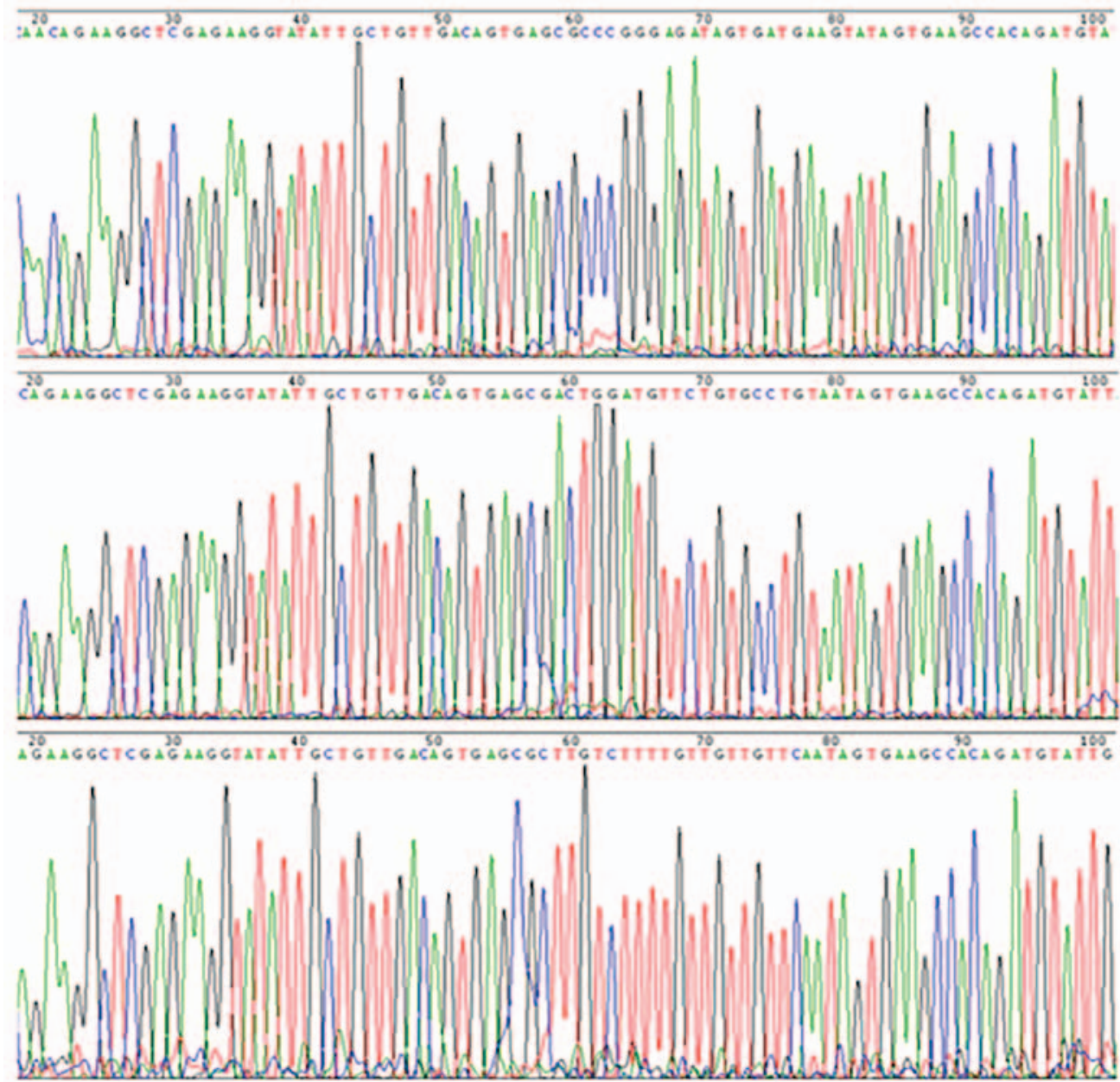

Figure 3. Sequencing results of the interference plasmids. The diagrams represent Bcl-2-sh1, Bcl-2-sh2 and Bcl-2-sh3 sequentially.

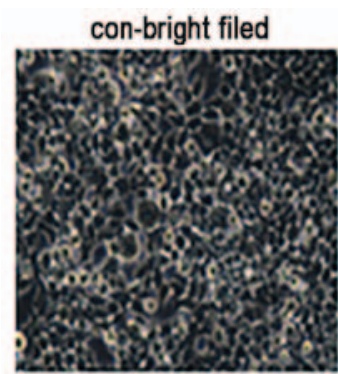

sh2-bright filed

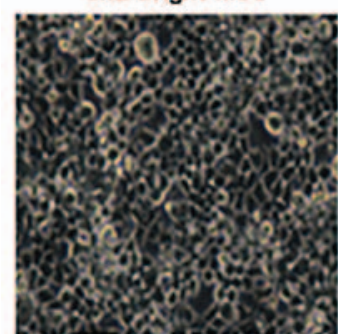

con-fluo. field

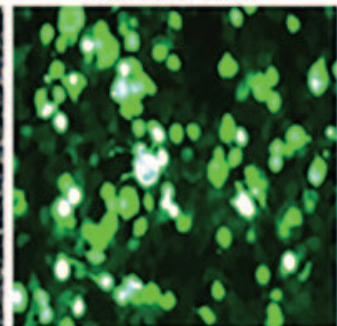

sh2-fluo. field

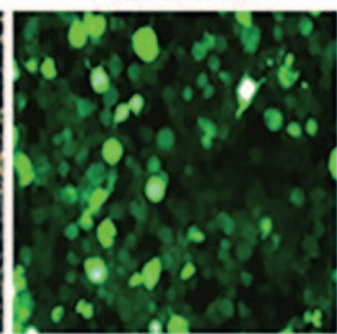

sh1-bright filed

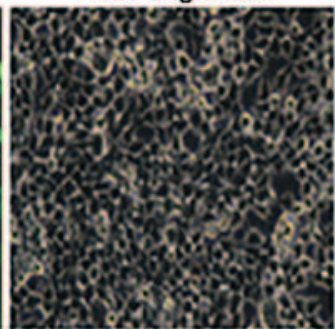

sh3-bright filed

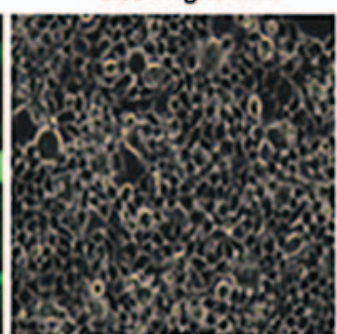

sh1-fluo. field

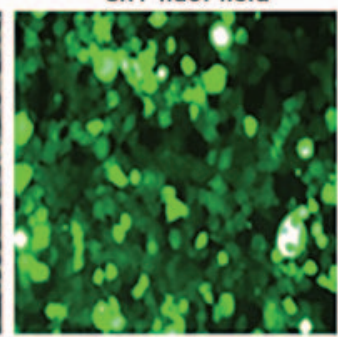

sh3-fluo. field

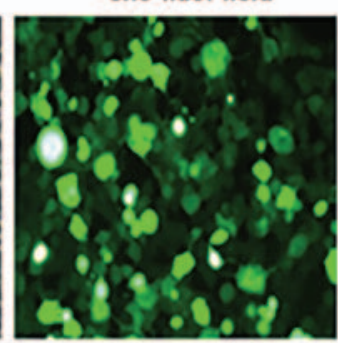

Figure 4. Fluorescence microscopy of SW982 cell morphology following transfection with the shRNA expression plasmids. con, control; shRNA, short hairpin RNA; fluo, fluorescence.

Bcl-2-sh3 plasmids significantly reduced Bcl-2 mRNA levels compared with the negative control group $(\mathrm{P}<0.05)$. The effect of Bcl-2-sh1 was the most pronounced (inhibition rate $>75 \%$ ). Therefore, $\mathrm{Bcl}-2$-sh1 was selected as the most effective interference sequence and was used for subsequent experiments (Fig. 5). 


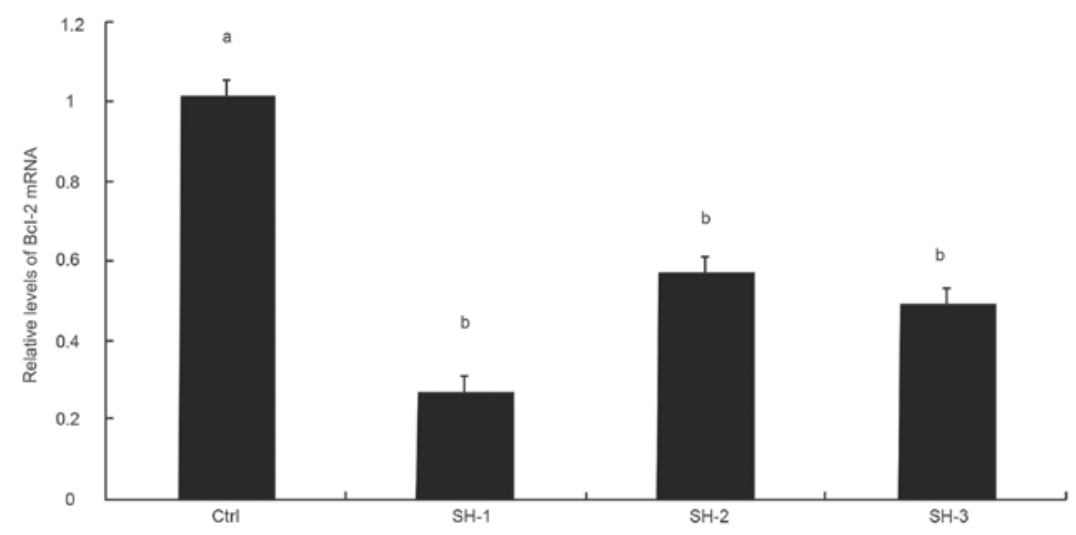

Figure 5. Expression levels of Bcl-2 mRNA in SW982 cells after transfection with the Bcl-2-shRNA expression plasmids. Sh1, Sh2, Sh3 and Ctrl indicate the cells transfected with Bcl-2-sh1, Bcl-2-sh2, Bcl-2-sh3 and negative-shRNA plasmids, respectively. Data are presented as the mean \pm standard deviation. Means with different superscript annotations are significantly different $(\mathrm{P}<0.01)$. Bars labeled with different letters $(\mathrm{a}, \mathrm{b})$ indicate statistically significant differences between groups; bars labeled with the same letters indicate no significant differences. Ctrl, control; sh, short hairpin.
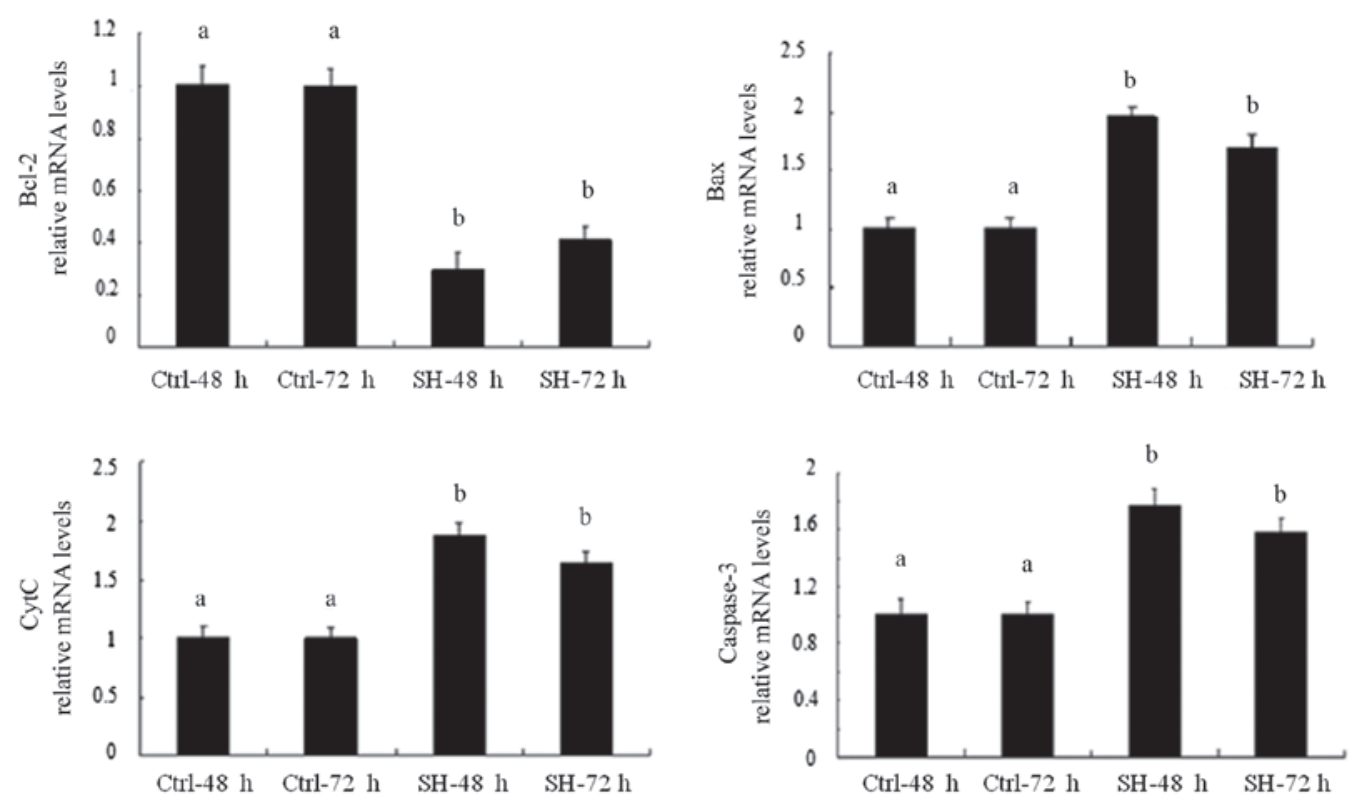

Figure 6. mRNA expression levels of mitochondrial pathway-associated genes in SW982 cells following transfection with Bcl-2-sh1. Sh- 48 h, Sh-72 h, Ctrl- 48 h and Ctrl-72 $\mathrm{h}$ indicate the cells transfected with Bcl-2-sh1 or negative-shRNA plasmid for 48 or $72 \mathrm{~h}$. Data are presented as the mean \pm standard deviation. Means with different superscript annotations are significantly different $(\mathrm{P}<0.01)$. Bars labeled with different letters $(\mathrm{a}, \mathrm{b})$ indicate statistically significant differences between groups; bars labeled with the same letters indicate no significant differences. Ctrl, control; sh, short hairpin.

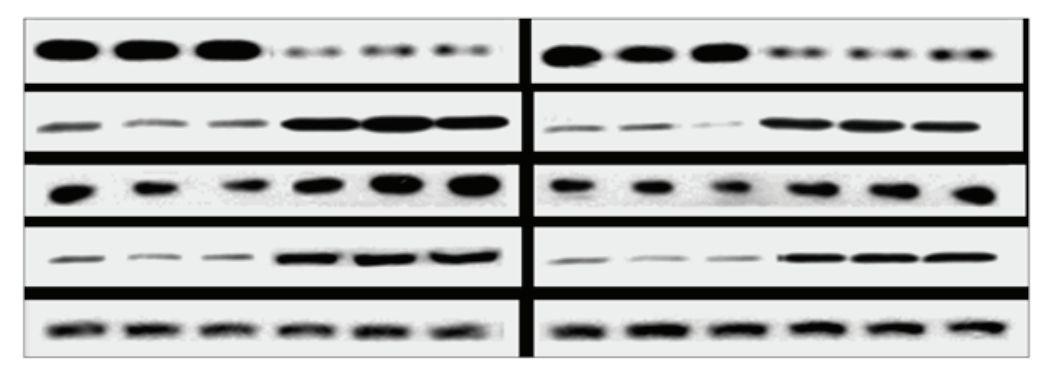

Figure 7. Protein expression levels of mitochondrial pathway components in SW982 cells following transfection with Bcl-2-sh1 after 48 and $72 \mathrm{~h}$.

Effect of Bcl-2-sh1 on the expression of mitochondrial pathway molecules. At 48 and $72 \mathrm{~h}$ following the transfection of Bcl-2-sh1 into SW982 cells, the mRNA and protein expression levels of Bcl-2, Bax, caspase-3, CytC were assessed. The results indicated that, compared with the control group, the expression of $\mathrm{Bcl}-2$ was significantly decreased, while on the contrary, Bax, CytC and Caspase-3 were significantly increased at the mRNA and protein levels; all differences were statistically significant $(\mathrm{P}<0.05)$. The effects of the Bcl-2 shRNA on the levels of Bcl-2, Bax, CytC and Caspase- 3 were more pronounced at $48 \mathrm{~h}$ than at $72 \mathrm{~h}$ post-transfection (Figs. 6 and 7). 


\section{Discussion}

Previous studies have demonstrated that the biological characteristics of synovial cells in patients with RA are markedly altered. Notably, significant enhancements in the proliferative rate and migratory ability of the cells have been observed. In addition, the Bcl-2 gene has been shown to be overexpressed in the synovial tissue and fibroblast-like synovial cells of RA patients, resulting in a deficiency in the apoptosis of inflammatory cells and an imbalance in immune homeostasis $(12,13)$. The human synovial sarcoma cell line SW982 possesses the characteristic of abnormal proliferation, similar to RA synovial tissue; therefore, its use for the study of RA has been recognized (14-16).

shRNA is a highly efficient gene-silencing molecule; compared with the traditional gene-silencing technologies, including gene knockout, negative mutation and antisense RNA, it has several advantages (17-19). Therefore, in the present study, the authors designed and synthesized Bcl-2 shRNA and transfected it into the SW982 cell line to observe its effects on the expression of mitochondrial apoptosis pathway genes, including Bcl-2, Bax, CytC and Caspase-3, in order to further study the relevance of this pathway to RA.

The reason for the selection of the Bcl-2 gene fragment for shRNA interference in the present study was the position and function of this gene in the mitochondrial pathway. Bcl-2 is an inhibitor of apoptosis and, as it is located upstream of the mitochondrial pathway, it is critical to the inhibition of this pathway. The Bcl-2 transmembrane protein contains two types of $\mathrm{Bcl}-2$ homology $(\mathrm{BH})$ domains: $\mathrm{BH} 1$ and $\mathrm{BH} 2$. At these regions, Bcl-2 and the pro-apoptotic gene Bax can form heterodimers or homodimers; this interaction is the basic mechanism by which Bcl-2 suppresses apoptosis $(20,21)$. The abnormal proliferation of synovial cells in patients with RA is associated with high expression of $\mathrm{Bcl}-2$. Therefore, the authors hypothesized that the construction of a Bcl-2-shRNA could directly activate the mitochondrial pathway at the source, enabling abnormal synovial cells to undergo apoptosis.

In the present study, bioinformatics methods were used in the design process, and the Bcl-2 mRNA sequences were obtained from GenBank. Sequences that were highly homologous with other genes were removed, and the GC content of the sequence was strictly limited to $35-55 \%$. For the loop structure in the shRNA template, in order to avoid the formation of a termination signal, the TTCAAGAGA sequence was selected. Three target sequences were designed, and the expressionvector method was used to prepare the shRNA (22-24). This method uses a plasmid with a resistance marker as a vector for transfection of the shRNA into cells, so as to achieve sustained suppression of target gene expression $(25,26)$. The results demonstrated that all three Bcl-2 shRNAs could inhibit the expression of Bcl-2 in SW982 cells, and the effect of Bcl-2-sh1 was the most obvious.

At 48 and $72 \mathrm{~h}$ following the transfection of Bcl-2-sh1 into SW982 cells, the results showed that the expression of Bcl-2 was significantly decreased, while the expression levels of Bax, CytC and Caspase-3 were significantly increased compared with those in the control group. Thus, the effectiveness of shRNA-mediated interference of Bcl-2 in human SW982 cells was confirmed, and it was demonstrated that this could indirectly promote the expression of other pro-apoptotic genes in the mitochondrial pathway. Since Bcl-2 and Bax exist in the form of homodimers or heterodimers, once the level of Bcl-2 is greater, $\mathrm{Bcl}-2 / \mathrm{Bcl}-2$ homodimers are formed, and apoptosis is inhibited. Bcl-2-shRNA inhibited the expression of the Bcl-2 gene and thereby changed the proportion of $\mathrm{Bcl}-2 / \mathrm{Bax}$, thus enhancing the expression of the pro-apoptotic gene Bax. Bax is a promoter of the mitochondrial pathway that promotes the release of $\mathrm{CytC}$, activates Caspase-3 and induces apoptosis of synoviocytes (27).

In summary, the interference effect of Bcl-2-sh1 on BCL-2 was more pronounced than that of the other two sequences, which demonstrated that, although many shRNA sequences may be designed for the same target gene, the interference effect might differ due to the different target sequences. With regard to the time-points, the interference effect of Bcl-2-sh1 was greater at $48 \mathrm{~h}$ than at $72 \mathrm{~h}$ post-transfection, indicating that the inhibitory effect of the shRNA was decreased over time; therefore, it is necessary to investigate ways of prolonging the silencing effect.

\section{Acknowledgements}

The present study was supported by grants from the Development Fund and Innovation Fund of Science and Technology (Medical and Health Projects) in Pudong New District of Shanghai, China (grant nos. PKJ2015-Y24 and 2015/05-2018/05).

\section{References}

1. Macintyre NJ, Muller ME, Webber CE and Adachi JD: The relationship between radial bone properties and disease activity and physical function in individuals with rheumatoid arthritis. Physiother Can 64: 284-291, 2012.

2. Navalho M, Resende C, Rodrigues AM, Pereira da Silva JA, Fonseca JE, Campos J and Canhão H: Bilateral evaluation of the hand and wrist in untreated early inflammatory arthritis: A comparative study of ultrasonography and magnetic resonance imaging. J Rheumatol 40: 1282-1292, 2013.

3. Krabben A, Abhishek A, Britsemmer K, Filer A, Huizinga TW, Raza K, van Schaardenburg DJ and van der Helm-van Mil AH: Risk of rheumatoid arthritis development in patients with unclassified arthritis according to the 2010 ACR/EULAR criteria for rheumatoid arthritis. Rheumatology (Oxford) 52: 1265-1270, 2013.

4. Shin SY, Katz P, Wallhagen M and Julian L: Cognitive impairment in persons with rheumatoid arthritis. Arthritis Care Res (Hoboken) 64: 1144-1150, 2012.

5. Li R, Yan G, Li Q, Sun H, Hu Y, Sun J and Xu B: MicroRNA-145 protects cardiomyocytes against hydrogen peroxide $\left(\mathrm{H}_{2} \mathrm{O}_{2}\right)$ induced apoptosis through targeting the mitochondria apoptotic pathway. PLoS One 7: 44907, 2012.

6. Mane SD, Thoh M, Sharma D, Sandur SK and Naidu KA: Ascorbyl stearate promotes apoptosis through intrinsic mitochondrial pathway in HeLa cancer cells. Anticancer Res 36: 6409-6417, 2016.

7. Zhang WD, Zhang Z, Zhang H, et al. Oxygen free radicals and mitochondrial signaling in oligospermia and asthenospermia. Mol Med Rep 10: 1875-1880, 2014.

8. Yan C, Kong D, Ge D, Zhang Y, Zhang X, Su C and Cao X: Mitomycin $\mathrm{C}$ induces apoptosis in rheumatoid arthritis fibroblast-like synoviocytes via a mitochondrial-mediated pathway. Cell Physiol Biochem 35: 1125-1136, 2015.

9. Liu L, Liu Y, Zhang X, Chen M, Wu H, Lin M, Zhan Y, Zhuang C, Lin $\mathrm{J}, \mathrm{Li} \mathrm{J}$, et al: Inhibiting cell migration and cell invasion by silencing the transcription factor ETS-1 in human bladder cancer. Oncotarget 7: 25125-25134, 2016. 
10. Li G, Zhang L, Liu J, Xiao T, Liu G, Wang J and Hou M: shRNA-mediated RPS15A silencing inhibits U937 acute myeloid leukemia cell proliferation and enhances apoptosis. Mol Med Rep 13: 4400-4406, 2016.

11. Livak KJ and Schmittgen TD: Analysis of relative gene expression data using real-time quantitative PCR and the 2(-Delta Delta C(T)) Method. Methods 25: 402-408, 2001.

12. Liu H, Yang Y, Cai X, Gao Y, Du J and Chen S: The effects of arctigenin on human rheumatoid arthritis fibroblast-like synoviocytes. Pharm Biol 53: 1118-1123, 2015.

13. Liu QS, Luo XY, Jiang H, Xing Y, Yang MH, Yuan GH, Tang Z and Wang H: Salvia miltiorrhiza injection restores apoptosis of fibroblast-like synoviocytes cultured with serum from patients with rheumatoid arthritis. Mol Med Rep 11: 1476-1482, 2015.

14. Sugiyama R, Agematsu K, Migita K, Nakayama J, Mokuda S, Ogura F, Haraikawa K, Okumura C, Suehiro S, Morikawa S, et al: Defect of suppression of inflammasome-independent interleukin-8 secretion from SW982 synovial sarcoma cells by familial Mediterranean fever-derived pyrin mutations. Mol Biol Rep 41: 545-553, 2014.

15. Beaulieu E, Green L, Elsby L, Alourfi Z, Morand EF, Ray DW and Donn R: Identification of a novel cell type-specific intronic enhancer of macrophage migration inhibitory factor (MIF) and its regulation by mithramycin. Clin Exp Immunol 163: 178-188, 2011.

16. Gupta A, Niger C, Buo AM, Eidelman ER, Chen RJ and Stains JP: Connexin43 enhances the expression of osteoarthritis-associated genes in synovial fibroblasts in culture. BMC Musculoskelet Disord 15: 425, 2014

17. Snead NM and Rossi JJ: RNA interference trigger variants: Getting the most out of RNA for RNA interference-based therapeutics. Nucleic Acid Ther 22: 139-146, 2012.

18. Nishioka N, Matsuoka T, Yashiro M. Hirakawa K, Olden K and Roberts JD: Plasminogen activator inhibitor 1 RNAi suppresses gastric cancer metastasis in vivo. Cancer Sci 103: 228-232, 2012.
19. Jung HS and Shin YK: The potential RNAi-based combination therapeutics. Arch Pharm Res 34: 1-2, 2011.

20. Stornaiuolo M, La Regina G, Passacantilli S, Grassia G, Coluccia A, La Pietra V, Giustiniano M, Cassese H, Di Maro S, Brancaccio D, et al: Structure-based lead optimization and biological evaluation of BAX direct activators as novel potential anticancer agents. J Med Chem 58: 2135-2148, 2015.

21. Siddiqui WA, Ahad A and Ahsan H: The mystery of BCL2 family: Bcl-2 proteins and apoptosis: an update. Arch Toxicol 89: 289-317, 2015.

22. Afonin KA, Grabow WW, Walker FM, Bindewald E, Dobrovolskaia MA, Shapiro BA and Jaeger L: Design and selfassembly of siRNA-functionalized RNA nanoparticles for use in automated nanomedicine. Nat Protoc 6: 2022-2034, 2011.

23. Mysara M, Garibaldi JM and Elhefnawi M: MysiRNA-designer: A workflow for efficient siRNA design. PLoS One 6: e25642, 2011.

24. Hefferon KL: Innovations in siRNA research: A technology comes of age. Recent Pat Antiinfect Drug Discov 5: 226-239, 2010.

25. Wang Y, Li Z, Han Y, Liang LH and Ji A: Nanoparticle-based delivery system for application of siRNA in vivo. Curr Drug Metab 11: 182-196, 2010.

26. Sarret P, Doré-Savard L and Beaudet N: Direct application of siRNA for in vivo pain research. Methods Mol Biol 623: 383-395, 2010.

27. Yuan S, Wu B, Yu Z, Fang J, Liang N, Zhou M, Huang C and Peng X: The mitochondrial and endoplasmic reticulum pathways involved in the apoptosis of bursa of Fabricius cells in broilers exposed to dietary aflatoxin $\mathrm{B}_{1}$. Oncotarget 7: 65295-65306, 2016.

28. Zhang W: Research of the pathogensis of oxygen free radicalsmitochondrion pathway in oligospermia and asthenospermia. Chinese doctoral dissertation full text database. May 2015. 